\title{
Las Reglas de Praga no son tan malas como parecen
}

\author{
Mario Reggiardo* \\ Eduardo Dibos*
}

Resumen. - Con cada vez mayor frecuencia se señala que el arbitraje internacional se ha convertido en un sistema de resolución de controversias oneroso, largo e ineficiente. Como intento de solucionar este problema, en 2018 se lanzaron las llamadas Reglas de Praga, un instrumento de soft law para conducir el arbitraje bajo la tradición del proceso civil inquisitivo. Así, los autores analizan las Reglas de Praga y las comparan con las Reglas IBA sobre Práctica de Prueba. Especialmente, revisan las reglas sobre exhibición de documentos, audiencias, testigos, peritos e inferencias. Afirman que, si bien las premisas en las que se fundan las Reglas de Praga no son del todo acertadas, podrían ser de utilidad para litigantes judiciales con poca experiencia en el arbitraje internacional. Consideran que variables exógenas al instrumento, como la destreza de los árbitros y la cultura legal de los abogados, incidirán en su éxito o fracaso.

\begin{abstract}
With increasing frequency, it is pointed out that international arbitration has become a costly, long and inefficient dispute resolution system. As an attempt to solve this problem, in 2018 the so-called Prague Rules were launched, an instrument of soft law to conduct arbitration under the tradition of inquisitive civil procedure. Thus, the authors analyze the Prague Rules and compare them with the IBA Rules on the Taking of Evidence. The authors review the rules on the display of documents, hearings, witnesses, experts e inferences. They suggest that, although the premises on which the Prague Rules are based are not entirely correct, they can be useful for judicial litigants with lesser experience in international arbitration. They consider that exogenous variables, such as the skills of the arbitrators and the legal culture of the lawyers, will affect their success or failure.
\end{abstract}

Palabras claves. - Arbitraje internacional - Procedimiento - Reglas de Praga - Reglas de la International Bar Association - Exhibición - Costos - Eficiencia.

Keywords. - International arbitration - Procedure - Prague Rules - Rules of the International Bar Association - Discovery - Costs - Efficiency.

* Abogado por la Pontificia Universidad Católica del Perú. Master en Derecho y Economía por la Universidad de Hamburgo. Profesor de Análisis Económico del Derecho y de Solución de Conflictos en la Universidad del Pacífico y en la Pontificia Universidad Católica del Perú. Socio en Payet Rey Cauvi Pérez. Miembro de la lista de árbitros de la Cámara de Comercio de Lima. mreggia@pucp.edu.pe

* Estudiante de séptimo ciclo de la Facultad de Derecho de la Universidad del Pacífico. Practicante en Bullard Falla Ezcurra +. Ganador del premio ALACDE Prize for Young Researchers en 2018 edibosf@gmail.com 


\section{Introducción ${ }^{1}$}

En diciembre del año 2018 fue presentada la versión final de las Reglas sobre la Tramitación eficiente de los Procedimientos en el Arbitraje Internacional, denominadas también Reglas de Praga (RDP)2. Este nuevo instrumento de soft law ha suscitado diversas polémicas en el mundo arbitral ${ }^{3}$. Las RDP empezaron a gestarse durante la IV Conferencia Anual de la Asociación de Arbitraje de Rusia, en la sesión titulada "La rampante americanización del arbitraje internacional: ¿es el momento adecuado para desarrollar reglas de evidencia inquisitivas?" 4 . Esta conferencia impulsó la preparación de un reglamento para la obtención de pruebas en el arbitraje internacional, inspirado en la tradición inquisitiva del proceso de los países del sistema romano-germánico (civil law). En su elaboración participaron sobre todo abogados europeos, la mayoría provenientes de ex repúblicas soviéticas y de Europa del Este. Los autores de las RDP tuvieron como objetivo contribuir a través de este nuevo instrumento a la eficiencia del arbitraje y así reducir su duración y costo ${ }^{5}$.

\section{El objetivo de las Reglas de Praga}

En el arbitraje internacional existe una gran variedad de reglas aplicables en la conducción del procedimiento ${ }^{6}$. Las RDP aparecen como una alternativa frente a las Reglas de la IBA sobre Práctica de Prueba en el Arbitraje Internacional (RIBA). Las RIBA -impulsadas por la International Bar Association- son desde 1999 el instrumento de soft law más recurrido y aceptado por las partes para guiar las actuaciones probatorias en arbitrajes internacionales y hasta locales ${ }^{7}$.

1 Los autores agradecen a Álvaro Cuba y Uber López por sus valiosos comentarios durante la preparación de este artículo.

2 "Prague rules premiere in Czech Republic". Global Arbitration Review (GAR). 7 de enero de 2019. Consultado el 10 de julio de 2019 en: https://globalarbitrationreview.com/article/1178408/prague-rules-premiere-in-czechrepublic.

3 Muchos de esos trabajos sirvieron en la preparación de este artículo y haremos referencia a ellos a lo largo del mismo.

4 Cuando decimos "rampante" estamos usando una traducción conservadora de la palabra "creeping" que se usó originalmente en idioma inglés y que se presta al juego de una interpretación peyorativa: "Creeping Americanization of international arbitration: is it the right time to develop inquisitorial rules of evidence?". PRAGUE RULES.. Is it time for a change?. 20 abril de 2017. Consultado el 11 de julio de 2019 en: http:/ / praguerules.com/news/is-it-time-for-a-change-/

5 Notas del Grupo de Trabajo encargado de la elaboración de las Reglas de Praga. Setiembre de 2018. Consultado el 10 de julio de 2019 en: http://praguerules.com/upload/medialibrary/b2e/b2e26123ac310b644b26d4cd11dc67d8.pdf

6 Por ejemplo, están el Reglamento de Arbitraje de la Cámara de Comercio Internacional (CCI), el Reglamento de Arbitraje de la Comisión de las Naciones Unidas para el Derecho Mercantil Internacional (CNUDMI-UNCITRAL), el Reglamento de Arbitraje de la Corte de Arbitraje Internacional de Londres (LCIA) o, en el plano local, el Reglamento de Arbitraje de la Cámara de Comercio de Lima (CCL), entre otros.

7 Según el International Arbitration Survey de 2015 elaborado por la Universidad de Queen Mary y White \& Case, las RIBA fueron consideradas las reglas de arbitraje más frecuentemente utilizadas (el 77\% de los encuestados las había puesto en práctica) y las que más alta valoración tuvieron entre ellos (el 69\% de los encuestados las consideró efectivas). Ver: Queen Mary University of London y White \& Case, 2015 International Arbitration Survey: Improvements and Innovations 
Las RDP establecen una serie de normas sobre la conducción de un arbitraje en general, no solo sobre la actividad probatoria, cuya principal característica es un tribunal bastante activo en la conducción del proceso. Las RDP presentan un procedimiento basado en una perspectiva inquisitiva, lo cual las diferencia de las RIBA $^{8}$. Buscan alejarse del arbitraje que funciona bajo el paradigma adversarial del common law, donde los juzgadores tienen un rol pasivo en el que se limitan a resolver las controversias en función de las herramientas que las partes les han proporcionado. En cambio, bajo el paradigma inquisitivo del civil law, el juzgador adopta un rol determinante en la obtención de pruebas y la determinación de la ley, como un agente activo de la búsqueda de la verdad en el proceso 9 .

Desde que fue publicado el primer borrador de las RDP en enero de 2018, se publicaron análisis que las comparaban con las RIBA ${ }^{10}$ y se alertó de un intento de la práctica rusa por cambiar el paradigma del arbitraje internacional ${ }^{11}$. Sin embargo, afirmar que las RDP representan al civil law mientras que las RIBA al common law, no es correcto. De hecho, desde su publicación en 1999, pasando por su actualización en 2010, las RIBA tuvieron como propósito tender puentes entre las prácticas del common y del civil law, al proporcionar un conjunto de normas que pueden ser usadas por profesionales de ambas tradiciones ${ }^{12}$.

Los propulsores de las RDP acusaron a la actividad probatoria, y en especial a la exhibición de documentos bajo el paradigma del litigio de los Estados Unidos de América, el discovery, como una de las principales causas por las cuales el arbitraje internacional puede ser largo y $\operatorname{costoso}^{13}$. Pero esta farragosa actividad probatoria no necesariamente es causada por dicha tradición jurídica, sino, entre otros factores, por una mala administración del caso por los árbitros y la paranoia del

in International Arbitration, 2015. Consultado el 11 de julio de 2019 en: https:// www.whitecase.com/sites/whitecase/files/files/download/publications/qmulinternational-arbitration-survey-2015_0.pdf

8 RESPONDEK, Andreas. "How civil law principles could help to make international arbitration proceedings more time and cost effective". En Singapore Law Gazzette, febrero 2017, p.34-35.

9 RAMOS ROMEU, Francisco. "Proceso inquisitorial y proceso adversarial: Orígenes y funcionamiento en 109 países". En Justicia: Revista de Derecho Procesal, núm 3-4,2008, p. 350.

10 MCILWRATH, Michael, “The Prague Rules: The Real Cultural War Isn't Over Civil vs Common Law", Kluwer Arbitration Blog, 12 de diciembre de 2018. Consultado el 11 de julio de 2019 en: http://arbitrationblog.kluwerarbitration.com/2018/12/12/the-prague-rules-the-real-culturalwar-isnt-over-civil-vs-common-law/

11 NEWMAN, Lawrence W. y ZASLOWSKY, David. “The Russians Are Coming, and They Want to Change How We Conduct International Arbitration", New York Law Journal. Consultado el 18 de mayo del 2019 en: https://www.law.com/newyorklawjournal/2018/05/23/the-russiansare-coming-and-they-want-to-change-how-we-conductinternationalarbitration/?slreturn $=20190418112130$

12 LINS SILVA E DUTRA, Luiza, "Taking of evidence in international arbitration: IBA Rules v. Prague Rules?" Bodenheimer Herzberg, 19 de diciembre de 2018. Consultado el 18 de mayo de 2019 en: https://www.changing-perspectives.legal/taking-of-evidence-in-internationalarbitration-iba-rules-v-prague-rules/

13 HENRIQUES, Duarte G. "The Prague Rules: Competitor, Alternative or Addition to the IBA Rules on the Taking of Evidence in International Arbitration?", Kluwer Arbitration Blog. 12 de diciembre de 2018. Consultado el 18 de mayo de 2019 en: https:// www.kluwerlawonline.com/abstract.php?area=Journals\&id=ASAB2018030 
debido proceso ${ }^{14}$ que lleva a muchos tribunales a aceptar la actuación de todo medio de prueba para reducir el riesgo de anulación de laudo. Yves Derains advierte que la fase de exhibición de documentos puede tornarse en una avalancha de documentos que hace ineficiente al arbitraje, lo cual debe ser evitado ${ }^{15}$.

Frente a la reacción internacional, que auguraba el poco éxito del proyecto, para la versión final las RDP redujeron su radicalidad. El borrador inicial señalaba explícitamente que las RDP nacían como respuesta a las RIBA y sus problemas. Pero al final se eliminó toda alusión directa a éstas últimas. Al monstruo se le cortaron las garras y se le puso una cadena. Finalmente mucho de lo que se intenta lograr con las RDP se puede alcanzar también con las RIBA. Por ello, en el preámbulo definitivo de las RDP se señala que "[L]a finalidad de las Reglas de Praga no es reemplazar las ya facilitadas por varias instituciones, sino que están diseñadas para complementar el procedimiento acordado por las partes"16. De igual manera, el co-presidente del grupo de trabajo a cargo de la elaboración de las RDP, Andrey Panev, señaló que las RDP no intentan reemplazar a las RIBA o posicionarse como la mejor manera de conducir un arbitraje ${ }^{17}$. Mas bien, remarca Panev, las RDP tienen la vocación de funcionar como un insumo adicional que puede ser o no usado y adoptado por las partes que estimen que un árbitro con mayor poder y proactividad conducirá su arbitraje de forma más eficiente ${ }^{18}$.

\section{Comparación temática entre las RDP y las Reglas IBA}

\section{i. Reunión Preliminar - Consulta sobre las cuestiones probatorias}

Las RDP y las RIBA coinciden en que una reunión o conferencia preliminar debe ser desarrollada para delinear las reglas de procedimiento que regirán al arbitraje. La RDP la denominan "Reunión preliminar", aunque es más descriptivo el nombre que se le da en la versión en inglés: Case Management Conference, término que se pudo traducir también como Conferencia de Gestión del Proceso. Las RIBA la

14 La paranoia del debido proceso o due process paranoia es la "renuencia de los tribunales a actuar con decisión en determinadas situaciones por temor a que el laudo arbitral sea cuestionado sobre la base de que una parte no tuvo la oportunidad de presentar su caso por completo". BERGER, Klaus Peter y JENSEN, Ole. "Due process paranoia and the procedural judgment rule: a safe harbor for procedural management decisions by international arbitrators". En Arbitration International, vol. 32, Edición 3, 2016.

15 DERAINS, Yves. "Towards Greater Efficiency in Document Production before Arbitral Tribunals-A Continental Viewpoint". En ICC Special Supplement 2006: Document Production in International Arbitration - International Chamber of Commerce, Paris: ICC International Chamber of Commerce, 2006, p. 85.

16 Preámbulo de las Reglas de Praga sobre la Tramitación Eficiente de los Procedimientos en Arbitraje Internacional. Consultado el 10 de julio de 2019 en: https://www.praguerules.com/upload/medialibrary/91c/91c0846151802b375541460b34e6b66 $\underline{\text { 3.pdf }}$

17 PANEV, Andrey, "Why the Prague Rules may be needed?" Thomson Reuters Arbitration Blog, 11 de octubre de 2018. Consultado el 10 de julio de 2019 en: http:/ / arbitrationblog.practicallaw.com/why-the-prague-rules-may-be-needed/

18 Idem. 
denominan "Consulta sobre cuestiones probatorias" y las disposiciones sobre ella se centran en el ámbito, tiempo y forma de la práctica probatoria.

Las RDP disponen que el tribunal convoque a la reunión preliminar apenas haya recibido los documentos del caso $^{19}$. En esta reunión se tratarán cuestiones procedimentales, como el cronograma del arbitraje. Los árbitros podrán comentar, pronunciarse y adelantar preliminarmente sus puntos de vista sobre materias del fondo de la controversia. El tribunal está facultado para compartir preliminarmente con las partes su entendimiento y opinión sobre la distribución de la carga de la prueba, el valor y la relevancia que le daría a éstas, el tipo de prueba que considera más apropiado, las pretensiones, los puntos controvertidos y los fundamentos de derecho. A nuestro juicio, la posibilidad de que el tribunal determine en ese momento la carga de la prueba y el tipo de medio de prueba que se necesita para que las partes acrediten los hechos podría resultar apresurado. El efecto de esta prematura e intensa actividad que pueden realizar los árbitros puede generar que aumente el costo de los honorarios arbitrales.

Las RIBA, por su parte, regulan lo que denominan "Consulta sobre cuestiones probatorias". A diferencia de las RDP, en donde es el tribunal quien impulsa las actuaciones en la reunión preliminar, en las RIBA el tribunal actúa como un observador a la espera que las partes se "consulten mutuamente" para que fijen ellas los aspectos probatorios del procedimiento ${ }^{20}$. Las instituciones sobre las cuales las partes podrán consultarse y pactar procedimientos son los dictámenes periciales, declaraciones testimoniales, audiencias, exhibición de documentos, confidencialidad, etc.

En contraposición a las RDP, las RIBA no disponen que los árbitros deban discutir preliminarmente las pretensiones planteadas por las partes ni los fundamentos de estas. El enfoque es procesal.

La práctica en estos casos suele ser flexible y depende de cada caso en concreto, a diferencia de las RDP que podrían ser apresuradas en cuanto a la oportunidad de la reunión previa. Pero, esto puede ser irrelevante en la práctica pues las partes podrían acordar con el tribunal durante su instalación, alejarse de reglas sobre la oportunidad de la conferencia y dejarla para después o incluso prescindir de esta.

El artículo 4 de las RDP $^{21}$ estipula que si una parte desea solicitar a la otra la presentación de material probatorio, deberá indicárselo al tribunal, como máximo,

19 Reglas de Praga, Artículo 2.1. “Después de recibir el expediente del caso, el tribunal arbitral celebrará una reunión preliminar, sin demora injustificada".

20 Reglas IBA, artículo 3.1. “Dentro del plazo fijado por el Tribunal Arbitral, cada Parte presentará a éste y a las demás Partes todos los Documentos que estén a su disposición y sobre los que base sus pretensiones, incluyendo Documentos públicos y de dominio público, exceptuando cualesquiera Documentos que ya hayan sido presentados por otra Parte".

21 Reglas de Praga, artículo 4.3. "No obstante, si una parte considera conveniente requerir a la parte contraria la aportación de determinados documentos, deberá indicárselo así al tribunal arbitral en la reunión preliminar, justificando los motivos por los que, en su opinión y en ese asunto específico, puede proceder la exhibición documental. [...]". 
con ocasión de la reunión preliminar. Si una parte dejó pasar aquella oportunidad, solamente podrá solicitar documentos en una fase posterior si concurren circunstancias excepcionales que serán determinadas por los árbitros ${ }^{22}$.

\section{ii. Peritos}

Los artículos 5 y 6 las RIBA ofrecen una extensa regulación sobre la actuación de los peritos designados por las partes y por el tribunal arbitral. Las RDP, al igual que las RIBA, admiten la designación de peritos a pedido de parte y a pedido del tribunal arbitral. Aquí la diferencia radica en que su regulación es mínima y acotada en comparación a lo que ofrecen las RIBA.

En las RIBA, si un perito designado por una de las partes no comparece en la audiencia de pruebas, el tribunal arbitral no podrá tener en cuenta su dictamen pericial23, salvo que en circunstancias excepcionales el tribunal decida lo contrario. El artículo 6.4 de las RDP admite la posibilidad que el perito de oficio sea convocado a audiencia para la ratificación de su informe ${ }^{24}$. Sin embargo, las RDP no supeditan la veracidad del informe del perito a su asistencia a la audiencia. Por lo tanto, en contraposición a las RIBA, el informe pericial seguirá siendo válido a pesar de que su autor no estuvo presente en la audiencia.

El artículo 5.2 de las RIBA regula una serie de contenidos mínimos que deberán tener los dictámenes periciales. Por ejemplo, los peritos deberán revelar su relación con cualquiera de las partes, su experiencia, declaración de hechos y opiniones e información utilizada para llegar a sus conclusiones, entre otros. En las RDP, mientras tanto, el contenido específico de los dictámenes periciales no es regulado a detalle.

En caso los peritos de parte hayan defendido posiciones distintas en sus informes, bajo las RIBA, el tribunal podrá solicitar a los peritos que se reúnan y deliberen acerca de los asuntos en donde haya existido una discrepancia con la finalidad de que este sea absuelto y así pueda presentarse un informe conjunto con sus acuerdos. De forma similar, según el artículo 6.7 de las RDP, también cabe la posibilidad que el tribunal ordene a los peritos a emitir un dictamen pericial conjunto en el que proporcionen al tribunal una lista de acuerdos y desacuerdos. Esto no solo alarga la duración del arbitraje, sino que posiblemente haga que se

22 Reglas de Praga, artículo 4.4. “Una parte podrá solicitar al tribunal arbitral que ordene la aportación documental durante una fase posterior del arbitraje solo si concurren circunstancias excepcionales. [...]".

23 Reglas IBA, artículo 5.5. "Si un Perito designado por la Parte cuya comparecencia ha sido solicitada de conformidad con el Artículo 8.1 no comparece a declarar en la Audiencia Probatoria sin justificación suficiente, el Tribunal Arbitral deberá descartar cualquier Dictamen Pericial de ese Perito Designado por la Parte relacionado con esa Audiencia Probatoria, salvo que, en circunstancias excepcionales, el Tribunal Arbitral decida lo contrario".

Reglas IBA, artículo 5.6. "Si la comparecencia de un Perito Designado por la Parte no ha sido solicitada de conformidad con el Artículo 8.1, se considerará que ninguna de las otras Partes ha aceptado la veracidad del contenido del Dictamen Pericial".

24 Reglas de Praga, artículo 6.4. “El perito podrá ser convocado para la ratificación de su informe en la audiencia, bien por el tribunal arbitral, de oficio, bien a solicitud de cualquiera de las partes". 
eleven los costos del servicio pericial. Si los abogados no intervienen, puede que los peritos se pongan de acuerdo, sobre todo si son peritos de prestigio e independientes. Si son peritos parcializados o los abogados intervienen, el costo de transacción se elevaría y se vería reducida la posibilidad de consensos. No obstante, y pese a que los peritos podrían cobrar las horas de trabajo adicionales, el beneficio representado por el consenso entre peritos podría ser enorme en tanto provea a los árbitros de indicios claros e identificables respecto de puntos controvertidos.

\section{iii. Exhibición de documentos}

Las RIBA regulan de modo exhaustivo la exhibición de documentos. Permiten su producción y buscan un equilibrio entre el discovery propio del common law y la exhibición limitada a documentos específicos propia del civil law. Los artículos 3.2 al 3.10 establecen la forma en que las partes deberán solicitar la exhibición, los requisitos para que esta sea válida, las objeciones que puede elaborar la contraparte, el razonamiento que deberá emplear el tribunal para resolver las objeciones, entre otros.

En cambio, las RDP regulan escasamente la exhibición de documentos. Siguiendo la vocación inquisitorial y de civil law en las cuales se fundan estas reglas, la exhibición de documentos a pedido de parte es desincentivada de manera deliberada en las RDP. Ello resalta a partir de su artículo 4.2: “Como regla general, se invita al tribunal arbitral y a las partes a evitar cualquier forma de exhibición documental, incluido el discovery electrónico" 25 .

En la mayoría de los procesos adversariales del common law la exhibición es amplia a efectos de revelar todos los hechos del conflicto, lo cual suele consumir mucho tiempo y dinero para las partes y sus abogados ${ }^{26}$.

Las RDP tienen la intención de reducir al mínimo indispensable la exhibición de documentos. El requisito de identificación de documentos concretos es rígido, a diferencia de la sola identificación de una categoría de documentos concretos en el artículo 3.3.(a)(ii) de las Reglas IBA27, que representa una solución intermedia entre las dos tradiciones ${ }^{28}$. A pesar de las ineficiencias que puede traer consigo, en

25 Reglas de Praga, artículo 4.2.

26 HANOTIAU, Bernard. "Document Production in International Arbitration: A Tentative Definition of 'Best Practices'. Opening Remarks". En ICC Special Supplement 2006: Document Production in International Arbitration - International Chamber of Commerce, Paris: ICC International Chamber of Commerce, 2006, p. 115.

27 Reglas IBA, artículo 3.3.a.ii. “Una solicitud de exhibición de documentos deberá contener: (ii) una descripción suficientemente detallada (incluyendo el asunto de que se trate) de la concreta y específica categoría de Documentos requeridos que razonablemente se crea que existen; en el caso de Documentos conservados en formato electrónico, la Parte solicitante puede ó el Tribunal Arbitral puede requerirle que proceda a, identificar archivos específicos, términos de búsqueda, individuos o cualquier otro medio de búsqueda para esos Documentos en una forma eficiente y económica".

28 Aunque para muchos litigantes del civil law, las RIBA sobre exhibición de documentos se acercan todavía al discovery del common law. En NEWMAN y ZASLOWSKY, Op. cit, p. 2. 
algunos casos la producción de documentos al que las partes tienen acceso contribuye a que el tribunal pueda tomar una decisión más informada y, por tanto, certera $^{29}$.

A pesar de ello, las RDP prácticamente niegan la importancia que tiene la exhibición de documentos en la resolución del arbitraje. El artículo 4.5 de las RDP dispone que una parte podrá solicitar al tribunal arbitral que requiera a la parte contraria la aportación de documentos $\operatorname{concretos}^{30}$. Solo podrá hacerlo si el tribunal considera que ello: i) es determinante para la resolución del caso; ii) no es de dominio público; y, iii) está en posesión o en control de la otra parte.

A diferencia de las RIBA, en donde las partes pueden dirigirse mutuamente solicitudes de exhibición de documentos de forma directa, bajo las RDP la solicitud de exhibición de documentos que elabora una parte debe de ser dirigida al tribunal primero. Recién cuando el tribunal se ha convencido que la exhibición es estrictamente necesaria y relevante para la aclaración de los puntos controvertidos, se efectúa la exhibición. Esto conlleva a que aumente el riesgo de error del tribunal, con el consecuente costo a la parte afectada. Por lo general, los tribunales no suelen tener información suficiente al inicio del arbitraje como para decidir ello correctamente. Si bien las reglas propuestas por las RDP en la práctica podrían producir ahorros en tiempo y costo de horas hombre en la producción de los documentos, el tribunal podría terminar denegando alguna prueba que de todas formas la contraparte pensaba exhibir. Es más eficiente que el tribunal se pronuncie solo cuando una parte se niega a producir el documento solicitado por su contraparte.

En tanto las RDP elevan el umbral que las partes deben satisfacer para solicitar un documento, se actuarán menos pruebas. Esto podría elevar los costos para las partes, aunque también para el tribunal. Por un lado, el tribunal, al dejar de tener acceso a ciertos documentos que podrían generarle una mayor convicción sobre los puntos controvertidos, se expone a un mayor riesgo de error en el laudo, lo que genera a su vez un daño para la parte afectada con el error. Por otro lado, se genera un contexto de información asimétrica entre las partes. Ello podría causar que estas se vean imposibilitadas de desplegar debidamente sus estrategias, lo cual aumenta las posibilidades que presenten escritos deficientes y que no generen convicción al tribunal.

29 KOCUR, Michal. "Why Civil Law lawyers do not need the Prague Rules". Kluwer Arbitration Blog. 18 de agosto de 2018. Consultado el 10 de julio de 2019 en: http://arbitrationblog.kluwerarbitration.com/2018/08/19/why-lawyers-from-civil-lawjurisdictions-do-not-need-the-prague-rules/

30 Reglas de Praga, artículo 4.5. “De conformidad con los Artículos 4.2 a 4.4, una parte podrá solicitar al tribunal arbitral que requiera a la parte contraria la aportación de un documento concreto: a) que sea determinante para la resolución del caso; b) que no sea de dominio público; y c) que esté en posesión o bajo el control de la parte contraria". 
En ese aspecto, son mayores los beneficios que generan las RIBA. Bajo su esquema, es posible elaborar una exhibición de documentos sin la participación activa de un tribunal, lo cual es deseable porque hace más dinámico el proceso. Solo se le pide al tribunal que participe si es que la contraparte ha presentado una objeción a la solicitud de exhibición. La ventaja de las RIBA es que como las partes desde un principio pueden solicitarse mutuamente pruebas, el tribunal tendrá menos que resolver, lo cual podría generar ahorro en tiempo y recursos. A pesar de que las parten suelen tener incentivos para hacer más célere este momento del arbitraje, existirán procesos en donde una de las partes querrá entrampar y alargar la práctica de pruebas.

Ayuda también la adopción de la tabla Redfern ${ }^{31}$. Por más que su uso no se encuentre previsto en las RIBA ni en las RDP, es muy común en los arbitrajes internacionales. La tabla Redfern es una gran ayuda para guiar y ordenar los procedimientos de las partes en un arbitraje de manera eficiente sin necesidad de recurrir a las restricciones de la RDP.

Bajo las RIBA, si una parte desea solicitar documentos a la otra podrá hacerlo solicitando una "categoría" de documentos que cree que razonablemente existen, tal como lo señala su artículo 3.a.II: "Una solicitud de exhibición de documentos deberá contener: [...] (ii) una descripción suficientemente detallada (incluyendo el asunto de que se trate) de la concreta y específica categoría de Documentos requeridos que razonablemente se crea que existen $[. . .]^{\prime \prime 32}$. Por ejemplo, solicitar una categoría de documentos sería pedir "todos aquellos correos electrónicos cursados entre la señora ' $X$ ' y la señorita ' $Y$ ' entre junio y agosto del 2018'.

La solicitud de una categoría de documentos incentiva el fishing expedition ${ }^{33}$. Las partes podrían solicitar gran cantidad de documentos con el fin de hacer incurrir a su contraparte en mayores costos y alargar el tiempo del arbitraje. Para evitar el fishing expedition, la parte que pide el documento debería tener la carga de la prueba: cuando no tiene un medio de prueba porque está en poder de la contraparte y le es necesario para probar los hechos, ahí debería estar facultada a pedir su exhibición. A nuestro juicio, la regla en cualquier instrumento debería ser que si la parte que pide los documentos no tiene la carga de la prueba, entonces no debería pedir el documento por que no necesita probar esos hechos para ganar el

31 La tabla o cronograma Redfern es un dispositivo utilizado comúnmente en la práctica comercial internacional que sirve para organizar solicitudes para la producción de documentos. Fue concebida como una forma de limitar la producción a los documentos críticos para el caso, y evitar los costos y retrasos asociados con el discovery del common law. Para una mayor explicación de su utilidad, dinámica actual y propuestas de mejora, ver: LUTRELL, Sam y HARRIS, Peter. “Reinventing the Redfern". En Journal of International Arbitration, vol. 33, núm. 4, 2016, p. 353-364.

32 Reglas IBA, artículo 3.3.a.ii

33 El fishing expedition es el término que define a todas aquellas solicitudes de producción de documentos elaboradas por las partes que no describen el contenido de los mismos ni identifican las alegaciones específicas que la parte solicitante pretende probar. Para mayor información al respecto ver: MARGHITOLA, Reto. "Interpretation of the IBA Rules". En Document Production in International Arbitration, Países Bajos: Kluwer Law International, 2015, pp. 61-64. 
arbitraje ${ }^{34}$. El costo de que la parte que no tiene la carga de la prueba genere la exhibición de documentos es alto frente al beneficio que se obtendría, ya que la inexistencia de la prueba igual no la perjudica directamente si es que su contraparte -la que tiene la carga de la prueba- no acreditó lo que necesita.

Por otro lado, en las RDP, el gran problema de los documentos "concretos" es que su solicitud parte de una premisa circular. Si la parte que lo pide tiene la carga de la prueba, puede que pierda el caso por la rigidez de esta disposición. No es razonable exigir que una parte detalle al tribunal el contenido del documento que desea conocer, porque posiblemente no lo conoce en detalle. Por el contrario, el interés que motiva a la parte a hacer el pedido es justamente conocer a detalle el documento que no se encuentra bajo su poder.

Contrario a lo señalado por el grupo de trabajo encargado de la redacción de las RDP, las RIBA sí establecen una serie de requisitos que limitan la exhibición de documentos y, por tanto, intentan evitar un perjuicio considerable a las partes. Las solicitudes tienen que cumplir con requisitos como la relevancia o utilidad del documento solicitado, impedimento legal, excesiva onerosidad, confidencialidad, etc. Las RDP no parecen buscar una mejora en el procedimiento de exhibición de documentos, sino más bien casi eliminarlo.

Por último, el énfasis de las RDP en el desincentivo para el discovery electrónico ${ }^{35}$ es absolutamente ajeno a la realidad en un entorno cada vez más tecnológico. Las actividades comerciales que originan los conflictos se alejan cada vez más de soportes materiales como el papel ${ }^{36}$, por lo que sigue creciendo la importancia de probar los hechos a partir de correos electrónicos, transacciones bancarias en línea, fotos digitales, mensajería instantánea etc. Además de anacrónica, esta disposición de las RDP puede generar a las partes un serio problema probatorio ${ }^{37}$, ya que muchos hechos de la actividad contemporánea no podrían ser probados, reduciendo las fuentes de prueba a opciones desfasadas e inexistentes.

\section{iv. Testigos}

Las RDP confían un rol proactivo y determinante al tribunal arbitral respecto de la toma de declaraciones de testigos. Bajo las RDP, un tribunal arbitral puede

34 ASHFORD, Peter. The IBA Rules on the Taking of Evidence in International Arbitration: A Guide. Reino Unido: Cambridge University Press, 2013, pp. 3-37. Citado por: MARGHITOLA, Reto. "Interpretation of the IBA Rules". En Document Production in International Arbitration, Países Bajos: Kluwer Law International, 2015, p. 56.

35 Reglas de Praga, artículo 4.2. "Como regla general, se invita al tribunal arbitral y a las partes a evitar cualquier método de exhibición documental, incluido el discovery electrónico".

36 IÑIGUEZ, Eduardo y CIRIANI, Carolina. "Arbitraje sin papel: llegó el momento", En CIAR GLOBAL, 2019. Consultado el 10 de julio de 2019 en: https:/ / ciarglobal.com/arbitraje-sin-papelllego-el-momento/

37 RIZZO AMARAL, Guilherme. Prague Rules v. IBA Rules and the Taking of Evidence in International Arbitration: Tilting at Windmills - Part II. 12 de diciembre de 2018. Consultado el 10 de julio de 2019 en: http:/ / arbitrationblog.kluwerarbitration.com/2018/07/06/prague-rulesv-iba-rules-taking-evidence-international-arbitration-tilting-windmills-part-ii/ 
determinar cuáles son los testigos que serán llamados a interrogatorio en audiencia. El artículo 5.3 de las RDP establece que los árbitros, antes o después de la declaración escrita del testigo, pueden decidir no llamarlo para la audiencia ${ }^{38}$, si considera que el testimonio que brindará es irrelevante, superfluo, reiterativo o por cualquier otro motivo no le ayudará a generarse convicción respecto de algún punto ${ }^{39}$. El artículo 5.7 señala, eso sí, que "si una parte insiste en la comparecencia" de un testigo sobre el que ya se aportó declaración escrita, el tribunal deberá citarlo salvo que existan razones justificadas para no hacerlo.

El interrogatorio será dirigido por los árbitros y éstos podrán rechazar aquellas preguntas que considere irrelevantes, redundantes o que no estén relacionadas con la controversia. Esta regla podría ser perjudicial para la dinámica de los interrogatorios a los testigos. Si bien en las RIBA los árbitros también pueden hacer preguntas a testigos en todo momento, las RDP van más allá y los árbitros podrían tener la confianza de interrumpir los interrogatorios con mucha discrecionalidad.

En contraposición, según el artículo 4.1 de las RIBA, son las partes, y no el tribunal las que determinan los testigos que llevarán a audiencia y que consideran que su testimonio contribuirá mejor a sus objetivos en el arbitraje.

Podría pensarse que una posible ventaja de las RDP en el sentido que el tribunal puede denegar la comparecencia de testigos en audiencia es que las partes, al saber que el tribunal tiene facultades de excluir a un testigo, se vean incentivadas a presentar solamente a los testigos más importantes. Pero ese mismo riesgo podría generar que las partes también presenten muchos testigos intentando que alguno de ellos sea aceptado, ya que sería poco probable que un tribunal rechace la actuación de todos. De hecho, esto es algo que ya se da en arbitrajes internacionales en donde confluyen los incentivos para, por un lado, evitar posibles costos de utilizar testigos sin relevancia, y por otro, nombrar demasiados testigos a manera de fishing.

Finalmente, más allá de las reglas que se utilicen, mucho va a depender de la cultura arbitral de los árbitros que las apliquen. También resultarán relevantes los incentivos de cada posible tribunal que se conforme, por ejemplo:

- Tribunal metódico, el cual apartará a testigos que considera que no ayudarán a resolver la controversia. Invertirá tiempo en fundamentar su decisión.

38 Reglas de Praga, artículo 5.3. “El tribunal arbitral podrá denegar la práctica del interrogatorio de determinados testigos durante la audiencia antes o después de la aportación al procedimiento de su declaración testifical escrita; especialmente, si considera que tal declaración es irrelevante, superflua, demasiado gravosa, reiterativa o, por cualquier otro motivo, innecesaria para la resolución de la controversia".

39 A manera de ejemplo, la Ley de Arbitraje Peruana también confiere amplias facultades probatorias a los árbitros, lo cual en términos genéricos, incluye a los testigos. Decreto Legislativo 1071, Ley de Arbitraje: “Artículo 43. Pruebas 1) El tribunal arbitral tiene la facultad para determinar de manera exclusiva la admisión, pertinencia, actuación y valor de las pruebas y para ordenar en cualquier momento la presentación o la actuación de las pruebas que estime necesarios. 2) El tribunal arbitral está facultado asimismo para prescindir motivadamente de las pruebas ofrecidas y no actuadas, según las circunstancias del caso". 
- Tribunal flojo pero con recursos, que no quiere audiencias prolongadas más allá de la utilidad de los testigos. Preferirá invertir recursos en fundamentar por qué deben excluirse testigos de las audiencias. Sabe que cuanto más corto el arbitraje, se vuelve más rentable su hora trabajada en el caso.

- Tribunal flojo pero sin recursos, preferirá no invertir recursos en fundamentar la irrelevancia del testimonio de un testigo. Tan solo lo escuchará, por más que el testimonio realmente nunca le haya parecido relevante.

- Tribunal adversarial, aquel que preferirá escuchar a todos los testigos y decidir luego la valoración que da a cada uno cuando lauden. Se da en el caso de árbitros cuyo prestigio se basa en que escuchan diligentemente, entre otros, a los testigos.

- Tribunal temeroso, este intentará evitar el riesgo de anulación del laudo bajo la causal de limitar el derecho de defensa de las partes. Este due process paranoia se ve traducido en que los árbitros aceptan prácticamente todas las pruebas y desestiman las oposiciones a su actuación, para reducir el riesgo de anulación del laudo por vulneración al derecho de defensa.

Las RIBA y las RDP presentan esquemas de relevancia de las declaraciones testimoniales escritas. Por el lado de las RIBA, según su texto, el tribunal arbitral podrá ordenar a las partes que presenten una declaración testimonial de su testigo para que este recién pueda comparecer en audiencia de pruebas ${ }^{40}$. $\mathrm{Al}$ margen de este requerimiento, generalmente son las partes quienes deciden qué y cuántos testigos comparecerán en la audiencia de pruebas. Igualmente, las RIBA supeditan la admisibilidad de una declaración testimonial a la comparecencia en audiencia de pruebas del testigo quien la emitió ${ }^{41}$. Si un testigo emite una declaración testimonial escrita pero no se presenta el día de la audiencia, el tribunal no podrá tomar en cuenta lo que dijo en su declaración inicial. De todas formas, esta regla contenida en el artículo 4.8 admite pacto en contrario; muchas veces las testimoniales escritas mantienen su procedencia y posibilidad de ser tomadas en cuenta en el laudo, así la contraparte no haya llamado al testigo para interrogatorio en audiencia.

En tanto las RIBA supeditan la admisibilidad de una declaración testimonial a la comparecencia en audiencia de pruebas del testigo, las partes al momento de estimar los costos de su defensa considerarán también el costo de llevar al testigo

40 Reglas IBA, artículo 4.4. “El Tribunal Arbitral podrá ordenar que cada Parte presente ante éste y ante las demás Partes, dentro de un plazo específico, una Declaración Testimonial de cada testigo en cuyo testimonio intente basarse, salvo que se trate de testigos cuyo testimonio se solicite de conformidad con los Artículos 4.9 o 4.10. Si se celebrasen Audiencias Probatorias sobre temas específicos $\mathrm{u}$ organizadas en fases (tales como jurisdicción, cuestiones preliminares, responsabilidad o daños y perjuicios), el Tribunal Arbitral o las Partes mediante acuerdo podrán programar la presentación de Declaraciones Testimoniales en forma separada para cada tema específico o fase".

41 Reglas IBA, artículo 4.7. "Si un testigo cuya comparecencia ha sido solicitada de conformidad con el Artículo 8.1 no compareciese a declarar en la Audiencia Probatoria sin justificación suficiente, el Tribunal Arbitral no tomará en cuenta ninguna Declaración Testimonial de ese testigo relacionada con tal Audiencia Probatoria, salvo que, en circunstancias excepcionales, el Tribunal Arbitral decida lo contrario". 
a declarar a la audiencia. El costo total por inclusión de testigo sería igual al costo por declaración escrita (horas de abogados, costo de recabar testimonio escrito más el costo por declaración en la audiencia, costo de preparación de testigos, entre otros). A ello se le podría sumar gastos por pasajes y viáticos si la audiencia es un lugar distinto al del domicilio del testigo. Para reducir el último componente del costo, es importante promover las videoconferencias, por ejemplo. Aunque el costo de ello es un eventual aumento del riesgo del error del tribunal al no poder estar atento a detalles reveladores de la comunicación gestual. Ello, por lo demás, es la paradoja de la minimización del costo administrativo de las reglas procesales, que suele tener como efecto el aumento del costo de error del juzgador ${ }^{42}$.

Consideramos que la exigencia de las RIBA de la sustentación obligatoria de todos los testigos en audiencia no es eficiente. Si una parte no llama a los testigos ofrecidos por la parte contraria, entonces asume el riesgo de que la pericia de la contraparte no se entienda. Aquella ponderación dependerá en si le conviene a una parte llamar o no al testigo contrario. Esta decisión debería ser parte de su libertad de actuación en el arbitraje.

Por su lado, las RDP no establecen disposiciones similares a las descritas en las RIBA. Si el tribunal considera que ha conocido suficientes argumentos con la declaración testimonial escrita, le bastaría y le es posible valorar tan solo ese contenido probatorio. Como señala su artículo 5.3, si un tribunal se ha generado convicción suficiente después de haber leído la declaración testimonial escrita de un testigo podrá decidir no llamarlo. En principio, esto podría parecer una disposición eficiente. Sin embargo, al utilizarla los tribunales se podrían estar privando de información valiosa que solamente puede ser rescatada durante las audiencias a través del contacto directo con las partes, sus abogados, peritos y testigos. La comunicación gestual, las respuestas espontáneas, las preguntas difíciles del abogado de la contraparte en el interrogatorio cruzado, tan solo por mencionar algunos ejemplos, son prácticas muy útiles que los tribunales tienen a su disposición para recabar valiosa información que no necesariamente fluye de la distante lectura de los testimonios escritos. A pesar de ello, Gary Born señala que el arbitraje internacional sigue tendiendo a basarse más en pruebas documentales (y declaraciones escritas de testigos) que en testimonios orales ${ }^{43}$.

Finalmente, ambas reglas comparten la característica que, en audiencia, los tribunales tienen la facultad expresa para dirigir los testimoniales y rechazar preguntas. En el artículo 8.244, las RIBA establecen el procedimiento general del

42 Cooter y Ulen señalan que el objetivo económico del derecho procesal es minimizar el costo social del proceso, que está determinado por la suma de los costos administrativos (Ca) y los costos de los errores (Ce): "mín CS = Ca + Ce". COOTER, Robert y ULEN, Thomas. Derecho y Economía. México: Fondo de Cultura Económica, 2008, p. 477.

43 BORN, Gary. International Commercial Arbitration. En Países Bajos: Kluwer Law International, Second Edition, 2014, pp. 2255-2256.

44 Reglas IBA, artículo 8.2. "El Tribunal Arbitral tendrá en todo momento pleno control sobre la Audiencia Probatoria. El Tribunal Arbitral podrá limitar o excluir cualquier pregunta, respuesta o comparecencia de un testigo, si considerase que dicha pregunta, respuesta o comparecencia es 
testimonio oral, dotando al tribunal de amplias facultades para dirigir la audiencia y prevé el interrogatorio cruzado a ser realizado por la defensa de las partes. Con las RDP el interrogatorio de cualquier testigo será dirigido y controlado por el tribunal. Al igual que las RIBA, los árbitros podrán rechazar preguntas que consideren irrelevantes o no estén relacionadas a la controversia. Siguiendo el criterio de eficiencia que persiguen las RDP, estas reglas desincentivan un interrogatorio cruzado extenso, lo cual no necesariamente implica que este se encuentre prohibido de realizarse. La paradoja con la máxima eficiencia es el riesgo de anulación de laudo por afectación al derecho de defensa de las partes. Para un tribunal que se guía por las RDP no siempre le será posible acortar la duración de los testimonios de testigos, puesto que los tribunales también desean mitigar las posibilidades de que su laudo sea anulado 45 .

En conclusión, las RIBA prefieren declaraciones de testigos al punto de que son prácticamente inevitables. Las RDP, en cambio, dan una menor importancia a las declaraciones de testigos en audiencia en tanto el tribunal arbitral tiene mayores incentivos para prescindir de ellas.

\section{v. Audiencias}

Las RDP indican expresamente que los árbitros y las partes deberán buscar que el caso sea resuelto solo con documentos ${ }^{46}$. Ello es una muestra de que se busca, en la medida de lo posible, evitar las audiencias donde se actúen pruebas testimoniales o periciales.

Bajo las RDP, en caso una de las partes solicite realizar una audiencia, se insta a que la misma sea conducida de la manera más eficiente posible. Incluso se recomienda el uso de herramientas audiovisuales para evitar que el tribunal y las partes incurran en mayores gastos al reunirse ${ }^{47}$. Por su parte, las RIBA favorecen que cada uno de los testigos y/o expertos testifique en persona. Se podría decir que las RDP parecieran, en este aspecto, una opción más eficiente. No obstante, el uso de herramientas tecnológicas que promueven la eficiencia dependerá, más allá del texto de la regla, de la práctica arbitral y el nivel de desarrollo de la sociedad de donde provengan las partes.

En las RDP, la regulación de la audiencia es escaza a comparación de las RIBA, que

irrelevante, insustancial, irrazonablemente gravosa, repetitiva o que de alguna otra manera se da alguno de los supuestos que justifique su objeción contemplados en el Artículo 9.2. Las preguntas y repreguntas efectuadas a un testigo durante su interrogatorio no deberán inducir irrazonablemente sus respuestas".

45 LINS SILVA E DUTRA, Op. cit.

46 Reglas de Praga, artículo 8.1 "Con la finalidad de promover la eficiencia y siempre que resulte adecuado para un asunto concreto, el tribunal arbitral y las partes deberán intentar resolver la controversia sobre la base de los documentos remitidos a las actuaciones".

47 Reglas de Praga, artículo 8.2. "Si una de las partes solicita la celebración de una audiencia o el tribunal considera procedente su celebración, las partes y el tribunal organizarán la misma de la manera más eficiente posible, incluyendo la posibilidad de limitar su duración y la utilización de video y comunicaciones electrónicas o telefónicas para evitar que los demás miembros del tribunal arbitral, partes y otros participantes incurran en gastos innecesarios de desplazamiento". 
sí tienen un tratamiento útil y detallado de los criterios de admisión de la prueba (artículo 9), así como del desarrollo de las audiencias probatorias (artículo 8). Al dedicar artículos a la regulación de criterios de admisibilidad de prueba y desarrollo de audiencia, las RIBA intentan potenciar, propagar y reducir costos de transacción de la partes en la organización de audiencias. Por su lado, la regulación de audiencia de las RDP es escaza y utiliza un solo artículo con dos numerales que contienen pautas genéricas (promoción de eficiencia y evitar celebración de audiencias) y no procedimientos específicos para la celebración de la audiencia.

Es cierto que los costos asociados a la celebración de audiencias, más aun en arbitrajes internacionales, puede representar un costo elevado en transporte y viáticos de abogados, testigos y peritos. A pesar de ello, es necesario ponderar los costos de la no realización de una audiencia y cómo esto puede llegar a afectar la generación de convicción de los árbitros. Puede resultar muy útil para el tribunal arbitral presenciar las comunicaciones gestuales de los testigos y expertos, las preguntas contextualizadas de los abogados y las respuestas espontáneas de los interrogados ${ }^{48}$. Ello solo se logra durante la audiencia en persona. De hecho, la prueba testimonial, si es confiable, tiene una especial fuerza persuasiva y permite probar hechos que no podrían demostrarse de otra manera ${ }^{49}$. Sin realización de audiencia presencial todos estos beneficios se perderían.

Al ser las RIBA bastante más exhaustivas en su regulación de los procedimientos vinculados a la audiencia de pruebas, estas reducen los costos de transacción en la medida que las partes no tienen que ponerse de acuerdo sobre las reglas del caso cuando se suscite la controversia. Esto último será cierto en la medida en que, para un caso en concreto, resulte inevitable la celebración de una audiencia. En ese sentido, resulta menos costoso para las partes haber pactado para su proceso las disposiciones que contienen los artículos 8 y 9 de las RIBA, que el artículo 8 de las RDP, ya que tendrán predictibilidad sobre cuáles serán las reglas que se apliquen a su arbitraje y no incurrirán en los costos de negociar un acuerdo posteriormente. En efecto, es probable que, para la celebración de una audiencia bajo las RDP, al ser sus reglas tan genéricas, las partes deberán incurrir en mayores costos de negociación para pactar un nuevo conjunto de reglas que direccione efectivamente su arbitraje. Pero, lo más probable, es que las partes que acepten arbitrar bajo las RDP, acepten las reglas que determine de oficio el tribunal.

\section{vi. Inferencias}

Las RDP estipulan que los árbitros podrán asumir conclusiones desfavorables para cualquiera de las partes cuando incumplan, sin un motivo justificado, las órdenes

48 LEW, Julian, MISTELIS, Loukas y KROLL, Stefan Michael. Comparative International Commercial Arbitration. Países Bajos: Kluwer Law International, 2003, p. 574.

49 PEJNOVIC, Milan y FERREYROS, Manuel. “Un testimonio vale más que mil escritos: el valor de la prueba testimonial": Enfoque Derecho, 31 de agosto de 2015. Consultado el 10 de julio de 2019 en: https://www.enfoquederecho.com/2015/08/31/un-testimonio-vale-mas-que-mil-escritosel-valor-de-la-prueba-testimonial/ 
o instrucciones del tribunal ${ }^{50}$. Según Rizzo Amaral, las conclusiones desfavorables o inferencias adversas pueden quedar definidas como la alternativa por la cual un tribunal arbitral llena con una interpretación razonable, propia e intuitiva, aquel vacío dejado por la evidencia que no fue presentada o producida por la parte quien tenía la carga de la prueba51.

Las RIBA admiten dos supuestos específicos para los cuales el tribunal podrá esbozar inferencias adversas: en la exhibición de documentos y ante la inasistencia de testigos a audiencias. Si una parte no presenta un documento o testimonio que ha sido requerido en una solicitud de exhibición u ordenado por el tribunal, este podrá inferir que ellos son contrarios a los intereses de la parte ${ }^{52}$. Igualmente, en caso un testigo haya sido llamado a comparecer en audiencia y este no asista injustificadamente, el tribunal arbitral no tomará en cuenta su declaración testimonial 53 .

La práctica de las inferencias adversas se encuentra más establecida en países del common law que en la tradición del civil law ${ }^{54}$. Es paradójico que los redactores de las RDP hayan rescatado un mecanismo de una tradición que cuestionan.

Las inferencias adversas sirven como un instrumento útil para llenar vacíos en el acervo de evidencia. Podrían, principalmente, posicionarse como una herramienta complementaria que dote al tribunal con elementos adicionales para generarse

50 Reglas de Praga, artículo 10. “Si una parte incumple las órdenes o las instrucciones del tribunal arbitral, sin justificación, el tribunal arbitral, cuando corresponda, podrá derivar conclusiones desfavorables para esa parte sobre ese aspecto o sobre su asunto".

51 RIZZO AMARAL, Guilherme. "Burden of Proof and Adverse Inferences in International Arbitration: Proposal for an Inference Chart". En Journal of International Arbitration, vol. 35, núm. 1, 2018, p. 9.

52 Reglas IBA, artículos 9.5. "Si una Parte no suministrare, sin explicación satisfactoria, un Documento requerido en una Solicitud de Exhibición de Documentos que ella no hubiera objetado en debido tiempo o no presentara un Documento que el Tribunal Arbitral hubiera ordenado aportar, el Tribunal Arbitral podrá inferir que ese Documento es contrario a los intereses de esa Parte".

Reglas IBA, artículos 9.5. "Si una Parte no cumpliere, sin explicación satisfactoria, con poner a disposición cualquier otra prueba relevante (incluyendo testimonios) solicitada por una Parte frente a la cual la Parte requerida no hubiera objetado en debido tiempo o no cumpliere con poner a disposición cualquier prueba, incluyendo testimonios, que el Tribunal Arbitral haya ordenado practicar, el Tribunal Arbitral podrá considerar que esa prueba es contraria a los intereses de esa Parte".

53 Reglas IBA, artículos 4.7 y 4.8. "Si un testigo cuya comparecencia ha sido solicitada de conformidad con el Artículo 8.1 no compareciese a declarar en la Audiencia Probatoria sin justificación suficiente, el Tribunal Arbitral no tomará en cuenta ninguna Declaración Testimonial de ese testigo relacionada con tal Audiencia Probatoria, salvo que, en circunstancias excepcionales, el Tribunal Arbitral decida lo contrario".

"Si la comparecencia de un testigo no ha sido solicitada de conformidad con el Artículo 8.1, se considerará que ninguna de las otras Partes ha aceptado la veracidad del contenido de la Declaración Testimonial".

54 GIEMZA, Wojceich. “The Principle of 'Adverse Inferences' In International Arbitration: Is Cour d'Appel Paving The Way For Civil Law Jurisdictions?”. En Mondaq, 2018. Consultado el 10 de julio de 2019

http://www.mondaq.com/x/610888/Arbitration+Dispute+Resolution/The+Principle+of+Ad verse+Inferences+In+International+Arbitration+Is+Cour+dAppel+Paving+The+Way+For+Civi $1+$ Law+Jurisdictions 
convicción sobre los puntos controvertidos y tomar posición para la elaboración de su laudo. Como sostiene Van Houtte, las inferencias adversas en el arbitraje constituyen un medio de evidencia indirecto, y solo tienen un peso limitado, pues tienen la vocación de encajar dentro y evaluarse junto con la totalidad de la evidencia que debe revisar el tribunal ${ }^{55}$. Entonces, una inferencia adversa no puede posicionarse como el fundamento principal de un laudo, sino más bien introducirse de forma accesoria o de soporte. En esa línea, recientemente la Corte de Apelaciones de Paris confirmó la aplicación de las inferencias adversas que un tribunal de la CCI utilizó en un arbitraje en donde las partes pactaron explícitamente su sujeción a las disposiciones de las RIBA ${ }^{56}$. La corte francesa remarcó que las inferencias hechas por el tribunal arbitral eran válidas solo en tanto tenían un carácter meramente complementario en la decisión, que de todas formas se basaba en la evidencia proporcionada por las partes.

Las inferencias adversas generan un trade-off que las partes deberán valorar caso por caso. Por un lado, la producción de documentos ante un pedido de exhibición puede llegar a ser una tarea pesada y costosa ${ }^{57}$. Pero para la parte que debe exhibir los documentos, ese costo puede ser bajo frente al costo de un fallo desfavorable incentivado por la inferencia adversa del tribunal. Las partes dejarán de entregar documentos cuando el costo esperado de la producción de documentos sea mayor al costo esperado de perder el caso por no entregar los documentos. Si producir la prueba cuesta, por decir, 3000 en horas hombre de la parte que buscará la documentación, la probabilidad de perder el caso por no entregarla, asumamos, es $5 \%$ y el laudo en contra sería de $1^{\prime} 000,000$. Entonces, $3000<0.05 \times 1^{\prime} 000,000$, con lo cual la parte correría el riesgo de no producir la prueba.

Las partes interiorizan los costos de presentar todos estos documentos. Sin embargo, si estas no cumplen con presentar los documentos requeridos bajo criterios de razonabilidad -sin perjuicio de que sean favorables o perjudiciales a su causa- se enfrentan a la posibilidad que el tribunal haga una inferencia adversa sobre las pruebas que fueron solicitadas y no presentadas. Un tribunal no hará una

55 VAN HOUTTE, Vera. “Adverse Inferences in International Arbitration”. Chapter 5., p. 214. En: GIOVANNINI, Teresa y MOURRE, Alexis (eds). "Written Evidence and Discovery in International Arbitration: New Issues and Tendencies". En Dossiers of the ICC Institute of World Business Law, Volume 6, 2009.

56 Cour d'Appel de Paris, Pôle 1 - Chambre 1, 28 February 2017, 15/06036. Para comentarios que suscitó aquella decisión ver: GIEMZA, Wojceich. "The Principle of 'Adverse Inferences' In International Arbitration: Is Cour d'Appel Paving The Way For Civil Law Jurisdictions?", En Mondaq, 2018. Consultado el 10 de julio de 2019 en http:// www.mondaq.com/x/610888/Arbitration+Dispute+Resolution/The+Principle+of+Ad verse+Inferences+In+International+Arbitration+Is+Cour+dAppel+Paving+The+Way+For+Civi

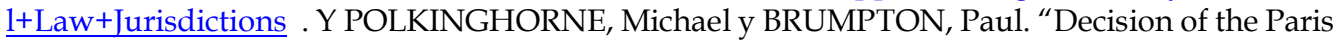
Court of Appeal on "adverse inferences" doctrine". En White \& Case, 2017. Consultado el 10 de julio de 2019 en https://www.whitecase.com/publications/alert/decision-paris-court-appealadverse-inferences-doctrine

57 Es discutible el hecho que la producción de documentos se posicione como la fase más costosa en el arbitraje. En arbitrajes en Estados Unidos de América la afirmación podría ser verdadera. Sin embargo, en el arbitraje internacional guiado por el paradigma de las RIBA, la fase de exhibición es acotada cuando es bien llevada por los árbitros. 
inferencia si, por ejemplo, no considera que el razonamiento que desarrollará es necesario o adecuado. Los árbitros deben ponderar el uso razonable de las inferencias junto con el respeto por el derecho de defensa las partes. ¿Qué debe pensar un tribunal al momento de elaborar inferencias adversas? Según Waincymer 58 , una inferencia adversa requiere una decisión del tribunal que tome en cuenta: (i) las razones por las cuales la evidencia no ha podido ser producida y (ii) la justificación propia del tribunal orientada a demostrar que la falta de producción probatoria amerita llevar a cabo una inferencia adversa. Las inferencias que haga un tribunal deben de ir en el mismo sentido de la evidencia que no se presentó, puesto que las inferencias deben de ser engranadas junto con las otras piezas que conforman las pruebas existentes.

Los tribunales no poseen una carta blanca para desplegar sus facultades de inferencia. Esta se ve limitada por el requerimiento de dar a las partes una oportunidad razonable para defenderse y presentar sus alegatos asociados a la producción de pruebas. Por ello, la inferencia representa un arma de doble filo para los tribunales. De constatarse los supuestos de los artículos 9 y 4 citados, los tribunales guiados por las RIBA podrán ejercer aquella facultad. Sin embargo, los incentivos para que el tribunal proceda a decidir sobre la base de una inferencia no siempre están dados. A mayor uso discrecional de las inferencias adversas, son mayores las posibilidades de una anulación de laudo. Por ello, los árbitros tienden a evitar confiar en inferencias adversas de forma expresa en sus laudos ${ }^{59}$. No obstante, siempre que esté apropiadamente delineada por un tribunal, una inferencia adversa no representará una violación del derecho de defensa de una parte ${ }^{60}$.

En el caso peruano, la probabilidad de anulación de un lado por el uso de inferencias es baja. Esto se debe a que la norma dispone que los jueces, ante un recurso de anulación de laudo, no pueden calificar la motivación ${ }^{61}$. En Perú, la única manera de anular en Perú, por ello, sería si el tribunal usó la inferencia adversa sin motivación alguna o de modo aparente.

Mientras que las RIBA limitan el poder de inferencias de los tribunales a la exhibición de documentos y a la inasistencia de testigos a las audiencias, las RDP no limitan los supuestos para su uso. Al no imponer límites o hacer distinción alguna sobre los temas en los que el tribunal puede hacer inferencias, las RDP incentivan a que los árbitros infieran con mayor frecuencia y libertad. Ello debería

58 WAINCYMER, Jeffrey. Procedure and Evidence in International Arbitration. Países Bajos: Kluwer Law International, 2012, p. 775.

59 MOREL DE WESTGAVER, Claire y ZINATULLINA, Ellina. “Will Adverse Inferences Help Make Document Production in International Arbitration More Efficient?". En Kluwer Arbitration Blog, 2017

60 WAINCYMER, Op. cit, p. 775

61 Decreto Legislativo 1071, Ley de Arbitraje. Artículo 62.2 - Recurso de anulación “El recurso se resuelve declarando la validez o la nulidad del laudo. Está prohibido bajo responsabilidad, pronunciarse sobre el fondo de la controversia o sobre el contenido de la decisión o calificar los criterios, motivaciones o interpretaciones expuestas por el tribunal arbitral". 
motivar a las partes a invertir mayores recursos en evitar incumplir alguna orden impuesta por el tribunal. Ahora, como la producción de documentos en las RDP es bastante limitada, el no cumplir en esos pocos casos puede justificar con mayor razón la inferencia. Si el tribunal ordena entregar un documento, es porque al ser preciso y detallado, podría generarse certeza de que existe y que se encuentra bajo poder de la otra parte y que ésta no lo quiere entregar.

\section{vii. Asistencia para un acuerdo transaccional}

De acuerdo a las RDP, los árbitros deberán asistir a las partes en la búsqueda de un acuerdo que ponga fin a la controversia, salvo que una de las partes lo objete ${ }^{62}$. Esto es más que nada una fórmula de buena voluntad para recordar a las partes que pueden transar. Una disposición como esta resulta de escasa utilidad en un arbitraje, salvo que estemos ante árbitros completamente inexpertos, faltos de criterio o que desconozcan que un arbitraje puede acabar también por acuerdo entre las partes.

Las RDP promueven que un árbitro actúe como mediador ${ }^{63}$. Con el acuerdo escrito de ambas partes, cualquiera de los árbitros podrá además actuar como tal. De no resultar exitosa la mediación, esta persona podrá volver a ser el árbitro en el caso si las partes lo desean. Esta podría ser una buena regla ya que el árbitro, entonces actuando como mediador, podrá, si la mediación fracasa y se regresa al arbitraje, haber analizado de cerca la disposición de las partes para transar, las intenciones que subyacen a sus pretensiones e incluso extraer conclusiones de las comunicaciones cursadas entre las partes y sus conductas en las reuniones celebradas durante esa fase. Esto podría proveer de mayores elementos de convicción al tribunal al momento de laudar. Una vez más, el éxito o fracaso de esta propuesta de las RDP radicará en la destreza del árbitro que actuaría como mediador. Las habilidades que se requieren para actuar acertadamente como mediador son distintas a los de un árbitro.

Las RIBA no incluyen mención expresa a la asistencia que prestaría un tribunal para que las partes transen. A pesar de ello, disposiciones como la 2.3(a) ${ }^{64}$ han sido comentadas favorablemente como un "paso importante para estimular un acuerdo

62 Reglas de Praga, artículo 9.1. “El tribunal arbitral podrá ayudar a las partes a alcanzar una solución transaccional de la controversia durante cualquier fase del arbitraje, salvo que concurra la objeción de una de las partes".

63 Reglas de Praga, artículo 9.2. "Cualquier componente del tribunal arbitral también podrá actuar como mediador para alcanzar una solución transaccional de la controversia, con el previo consentimiento escrito de todas las partes".

64 Reglas IBA, artículo 2.3(a). "Se invita al Tribunal Arbitral a identificar ante las Partes, tan pronto como lo considere pertinente, cualquier cuestión: (a) que el Tribunal Arbitral pueda considerar relevante para el caso y sustancial para su resolución". 
entre las partes, en la medida que a través de dicha evaluación temprana se dan cuenta de sus posibilidades de tener éxito en el arbitraje" 65 .

De todas formas, y como ocurre en la práctica, las partes son libres de llegar a un acuerdo en cualquier fase del arbitraje, lo cual obviamente no está prohibido por estas reglas. Es importante que el tribunal tenga una actitud favorable a suspender el caso si las partes lo solicitan, pero no se le puede exigir que tenga una actitud proactiva en ese sentido porque el rol del árbitro es distinto al de mediador.

\section{viii. Iura novit curia}

Las RDP expresamente permiten el iura novit curia en su artículo 7.266. El tribunal arbitral podrá sustentar sus decisiones en normas legales que no hayan sido alegadas por las partes. Sin embargo, para el uso del iura novit curia se exige que las partes sean previamente consultadas sobre dicha aplicación. Igual consideración con las partes deberá tener el tribunal si utilizará doctrina o jurisprudencia no alegada (denominados antecedentes legales o legal authorities en las RDP). La facultad del iura novit curia por parte de los juzgadores es un signo distintivo clásico de la tradición civil e inquisitorial, en donde se entiende que los jueces tienen el deber de conocer y aplicar las normas en resguardo del ordenamiento jurídico. En el sistema adversarial del common law, en cambio, el tribunal suele decidir solo entre las posiciones que las partes le han planteado ${ }^{67}$. Esta diferencia de visión entre las tradiciones de derecho civil y common law es una de las distinciones más significativas entre ambos sistemas ${ }^{6}$.

Esta facultad no está prevista en las RIBA, lo cual no implica que esté prohibida tampoco.

Ahora bien, teniendo en cuenta que el iura novit curia es una facultad usada en sede judicial y en tradiciones civiles, su aplicación en el arbitraje internacional no es siempre aceptada en la práctica y la doctrina. Para Blackaby y Chirinos ${ }^{69}$, por ejemplo, la aplicación del iura novit curia no resulta apropiada en el arbitraje

65 BERGER, Klaus Peter y JENSEN, Ole. "The Arbitrator's Mandate to Facilitate Settlement", En International Commercial Arbitration Review - Association of Researchers in International Private and Comparative Law, núm. 1, 2017, p. 62.

66 Reglas de Praga, artículo 7.2. “Sin embargo, el tribunal arbitral podrá aplicar disposiciones legales que no hayan sido invocadas por las partes si así lo estima necesario, incluyendo, entre otras, normas imperativas. En estos supuestos, el tribunal arbitral recabará el parecer de las partes sobre las normas legales que intente aplicar. El tribunal arbitral también podrá apoyarse en antecedentes legales -aunque no hayan sido citados por las partes- si se refieren a disposiciones legales alegadas por las partes y siempre que las partes haya tenido oportunidad de expresar su parecer sobre su contenido".

67 RESPONDEK, Andreas. "How civil law principles could help to make international arbitration proceedings more time and cost effective". En Singapore Law Gazzette, febrero 2017, p. 34.

68 MANN, Frederick., "Fusion of the Legal Profession". En Law Quarterly Review, vol. 93, núm. 3, 1977, pp. 367, 375. Citado por WAINCYMER, Jeffrey. "Procedure and Evidence in International Arbitration". En Kluwer Law International, 2012, p. 1064.

69 BLACKABY, Nigel y CHIRINOS, Ricardo. "Consideraciones sobre la aplicación del principio iura novit curia en el arbitraje comercial internacional". En Anuario Colombiano de Derecho Internacional - ACDI, vol. 6, 2013. p. 82. 
comercial internacional, al menos en la misma forma en que dicho principio es utilizado por los jueces al momento de dictar su sentencia conforme a su derecho nacional. La razón de ello es que el principio no puede ser trasladado al campo del arbitraje comercial internacional de forma automática y sin sufrir transformación alguna dadas las diferencias estructurales entre el arbitraje y el proceso judicial. No obstante esa posición, los autores consideran que el iura novit curia sí podría ser usado en casos excepcionales y guardando estricta observancia del derecho de defensa de las partes.

Otros como Newcombe y Paradell señalan que, en principio, sí podría utilizarse el iura novit curia en el arbitraje guardando importantes reparos: "no puede extenderse [el iura novit curia] hasta el punto en que el tribunal deba complementar argumentos no alegados, pretensiones no presentadas o defensas no señaladas"70. Por su parte, Alberti ${ }^{71}$ al igual que Waincymer ${ }^{72}$, señalan que si bien la presencia del iura novit curia en el arbitraje internacional no es pacífica, su aplicación existe y difiere entre cada país, y depende de su engranaje con los principios del arbitraje y los derechos de las partes en cada sistema. El último autor señala que el iura novit curia es más comúnmente utilizado en arbitrajes en donde la tradición civil es la reinante, mientras que en los países del common law su uso es mucho más resistido.

En nuestra opinión, en principio, en un arbitraje no debería aplicarse el iura novit curia porque los árbitros son contratados para que escojan entre las posiciones que las partes les plantean. En el arbitraje comercial los árbitros no tienen el deber de proteger la aplicación armónica de las leyes nacionales ${ }^{73}$, deber que sí tienen los jueces. Eventualmente, el iura novit curia podría aplicarse para sustentar alguna norma adicional que refuerce una posición no primaria de las partes. Es decir, un tribunal, a través del uso del iura novit curia, no debería poder sustentar un cambio de la calificación jurídica de una pretensión principal de alguna parte. Por ejemplo, si las partes están discutiendo sobre las consecuencias de un contrato de arrendamiento mal regulado, en el laudo los árbitros no pueden fallar aplicando normas de comodato porque consideren que es el contrato aplicable, mucho menos si los árbitros no dieron antes a las partes la oportunidad de pronunciarse sobre esa hipótesis.

70 NEWCOMBE, Andrew y PARADELL, Luis. Law and Practice of Investment Treaties: Standards of Treatment. Países Bajos: Kluwer Law International, 2009, p. 90.

71 ALBERTI, Christian. "Iura Novit Curia in International Commercial Arbitration: How Much Justice Do You Want?. En KROLL Stefan Michael, MISTELIS, Loukas, et. al. (eds), International Arbitration and International Commercial Law: Synergy, Convergence and Evolution, Países Bajos: Kluwer Law International, 2011, p. 28

72 WAINCYMER, Jeffrey. Procedure and Evidence in International Arbitration. Países Bajos: Kluwer Law International, 2012, p. 1067.

73 A diferencia del arbitraje de contrataciones con el Estado peruano., donde expresamente el artículo 45.10 del TUO de la Ley $\mathrm{N}^{\circ} 30225$, Ley de Contrataciones del Estado expresa lo siguiente: “45.10 Las controversias se resuelven mediante la aplicación de la Constitución Política del Perú, de la presente norma y su reglamento, así como de las normas de derecho público y las de derecho privado; manteniendo obligatoriamente este orden de preferencia en la aplicación del derecho. Esta disposición es de orden público". 


\section{Conclusiones}

Las RDP parten de la premisa que un tribunal arbitral activo en la determinación de los hechos del caso vuelve más eficiente el arbitraje. Ello no es siempre cierto. En las RDP se confunde el rol activo del tribunal en la dirección del arbitraje y en la clarificación de los hechos. Lo primero es deseable ya que son los árbitros quienes tienen la mejor posición para conducir las actuaciones arbitrales. Pero lo segundo no lo es. Son las partes quienes conocen mejor los hechos. Cada una de ellas se esforzará por presentar todos los medios de prueba que puedan beneficiarla. Los árbitros suelen conocer recién los detalles de la controversia con el procedimiento algo más avanzado ${ }^{74}$.

Las RDP consideran que la ineficiencia de los arbitrajes solo se debe al sistema de reglas de práctica de pruebas derivado del common law. Pero existen otros factores que también las causan. Las RDP solo permitirán que si las partes se sienten cómodas conduciendo su arbitraje bajo un procedimiento inquisitivo, exista un reglamento que lo facilite. En todo caso, el éxito de las RDP dependerá de la capacidad y experiencia de los árbitros. Otorgar un rol más activo a un buen árbitro puede contribuir a tener un arbitraje eficiente. No obstante, si las mismas facultades son otorgadas a árbitros inexpertos o erráticos, los efectos pueden ser perjudiciales y se pone en riesgo el derecho de defensa de las partes.

Es bueno que existan las RDP como una opción entre las reglas disponibles para seguir un arbitraje. Para arbitrajes simples y abogados ajenos a la práctica de arbitrajes internacionales sofisticados, las RDP pueden ser de utilidad frente a las RIBA $^{75}$. Incluso abogados que suelen litigar solo en cortes judiciales y no tienen mucha experiencia arbitral, podrían sentirse cómodos litigando bajo las RDP. Si las partes no se manejan bajo los estándares de las RIBA u otros reglamentos adversariales del arbitraje internacional, y prefieren una dirección del proceso más paternalista o autoritaria76, las RDP representan una manera alternativa de litigar, delegando en los árbitros un férreo control de la actividad probatoria y de la dirección del arbitraje en general.

KOCUR, Op. cit.

5 MCILWRATH, Michael, “The Prague Rules: The Real Cultural War Isn't Over Civil vs Common Law", Kluwer Arbitration Blog, 12 de diciembre de 2018. Consultado el 11 de julio de 2019 en: http://arbitrationblog.kluwerarbitration.com/2018/12/12/the-prague-rules-the-real-culturalwar-isnt-over-civil-vs-common-law/

76 Con "autoritario" hablamos de un tribunal con mucho poder para decidir aspectos que en el arbitraje usualmente se tratan de modo más flexible y con soluciones de mercado. Ver también NEWMAN y ZASLOWSKY, Op. cit, p. 6. 